\title{
Coordinated control for voltage regulation of distribution network voltage regulation by distributed energy storage systems
}

\author{
Delong Zhang ${ }^{1}$, Jianlin $\mathrm{Li}^{\mathrm{i}^{*}}$ and Dong Hui
}

\begin{abstract}
With more and more distributed photovoltaic (PV) plants access to the distribution system, whose structure is changing and becoming an active network. The traditional methods of voltage regulation may hardly adapt to this new situation. To address this problem, this paper presents a coordinated control method of distributed energy storage systems (DESSs) for voltage regulation in a distribution network. The influence of the voltage caused by the PV plant is analyzed in a simple distribution feeder at first. The voltage regulation areas corresponding to DESSs are divided by calculating and comparing the voltage sensitivity matrix. Then, a coordinated voltage control strategy is proposed for the DESSs. Finally, the simulation results of the IEEE 33-bus radial distribution network verify the effectiveness of the proposed coordinated control method.
\end{abstract}

Keywords: Distributed energy storage, Voltage regulation, Voltage sensitivity, Coordinated control, Photovoltaic (PV) plants

\section{Introduction}

In the past decade, the development of wind power and PV generation is very fast because of their technical and environmental advantages. They also bring several new challenges to power system. With the improvement of permeability of PV generation in the distribution network, the traditional distribution network becomes an active network, whose voltage bus connected PV generation is higher than other bus [1].

The traditional distribution network has multiple voltage regulation methods, such as on-load tap changer (OLTC) [2], reactive power regulator [3]. However, these methods cannot always regulate the voltage flexibly and effectively, because the voltage is different from the network without PV. The voltage of one node is regulated to the normal range, the others maybe exceed caused by the action of regulator. Moreover, the frequent transformation of the power grid will be very uneconomical. There are usually two ways to adjust voltage using a

\footnotetext{
* Correspondence: dkylj|@163.com

${ }^{2}$ State Key Laboratory of Operation and Control of Renewable Energy \& Storage System, China Electric Power Research Institute, Haidian District, Beijing 100192, China

Full list of author information is available at the end of the article
}

distributed generation (DG). One is to increase the reserve capacity of the distributed power supply, which obviously cannot maximize the utilization rate of renewable energy. The other is to apply inverter to generate reactive power to regulate voltage, which will increase inverter efficiency. In [4], the authors proposed a voltage regulation method by adjusting the inverter DG injected active power. In [5], the voltage regulation criterion of DG connected to the distribution network is proposed, and the capacity and position of reactive power compensation are determined accordingly. In [6, 7], it is found that DG should reduce the active output according to the actual voltage of the grid. Obviously, this method is not conducive to the full play of DG. In [8], a coordinated control method based on DGs and OLTC voltage regulation is proposed, which divides the DGs and OLTC adjustment area and controls its processing order to reduce the operation times of the on-load tap changer.

The DESS is usually installed with distributed PV plant. Expect for the normal operation, DESS can be used to regulate the voltage. It can not only improve the utilization of PV power, but also improve the voltage quality of power system $[9,10]$. Distribution network 
contains a number of DESSs, if they are in a chaotic state, it cannot play the role in voltage regulation. Therefore, the main challenge using DESSs to regulate voltage is how to coordinate DESSs reasonably and determine the output power $[11,12]$.

In [13], the authors propose a coordinated control method of DESSs, which includes distributed and localized controls. The consensus algorithm is used to regulate the feeder voltage, while the localized control regulates the state of charge $(\mathrm{SoC})$ of each ESS within the desired SoC range. In [14], the former one is voltage regulation in terms of their installed capacity and the latter one modifies the BESs performance according to their SoC. These papers mainly focus on the voltage regulation of low voltage distribution network that the area is small. They are not suitable for large area medium voltage distribution network.

In this paper, a coordinated control method using distributed energy storage is proposed for adjusting the voltage of a medium voltage distribution network. Firstly, in the three-node simple system, the voltage of system with and without PV plant is compared. The influence caused by PV plant is analyzed. Secondly, a method is proposed to divide the voltage regulation area by calculating and comparing the voltage sensitivity matrix. On this basis, coordinated control sequence of DESSs and the model of the energy storage system is established. Finally, the simulations are carried out on the IEEE 33-node system.

The paper is organized as follows. The theory of voltage deviations in the distribution feeders with high penetration of PV cells is explained in section 2. The proposed coordinated control strategy of distribution network voltage regulation is presented in section 3 . Section 4 introduces the IEEE 33-bus test distribution system and presents simulation results. Finally, Section 5 concludes the paper.

\subsection{Problem description}

In the distribution network, the voltage is generally reduced with the extension of the feeder. The access of distributed PV generations changes this situation. In this part, a simple three-node system is used to illustrate the problem.
As shown in Fig. $1, a, b, c$ are three nodes. $Z_{1}$ and $Z_{2}$ are the impedance of line 1 and line 2 respectively. $S_{1^{\prime}}$ and $S_{1}$ " are the power of the start and the end of line 1 respectively. $S_{2^{\prime}}$ and $S_{2}$ the power at the start and end of line 2 respectively. $S_{b}$ and $S_{c}$ are the load of node $b$ and node $c$ respectively.

In the power system without PV generation, some equations can be obtained according to the simple power flow calculation method.

The power at the end of line 2 is

$$
S_{2 "}=S_{\mathrm{c}}
$$

The power loss of line 2 is

$$
\Delta S_{2}=\left(\frac{S_{2^{\prime \prime}}}{V_{N}}\right)^{2}\left(R_{2}+j X_{2}\right)
$$

The power at the start of line 2 is

$$
S_{2^{\prime}}=S^{\prime \prime}+\Delta S_{2}
$$

The power at the end of line 1 is

$$
S_{2^{\prime \prime}}=S_{b}+S_{2^{\prime}}
$$

The power loss of line 1 is

$$
\Delta S_{1}=\left(\frac{S_{1^{\prime \prime}}}{V_{N}}\right)^{2}\left(R_{1}+j X_{1}\right)
$$

The power at the start of line 1 is

$$
S_{1^{\prime}}=S_{1^{\prime \prime}}+\Delta S_{1}
$$

The loss of voltage longitudinal component of line 1 is

$$
\begin{aligned}
& \Delta V_{1}=\left(P_{1^{\prime}} R_{1}+Q_{1^{\prime}} X_{1}\right) / V_{N} \\
& V_{b}=V_{a}-\Delta V_{1}
\end{aligned}
$$

The loss of voltage longitudinal component of line 2 is

$$
\begin{aligned}
& \Delta V_{2}=\left(P_{2^{\prime}} R_{2}+Q_{2^{\prime}} X_{2}\right) / V_{N} \\
& V_{c}=V_{b}-\Delta V_{2}
\end{aligned}
$$

It is assumed that the voltages of node $b, c$ have meet the error requirements. It is obviously that the voltage of node $c$ is smaller than $b, b$ is smaller than $a$.

When adding the PV station on node $c$, in order to clarify the problem more clearly, we assume that the

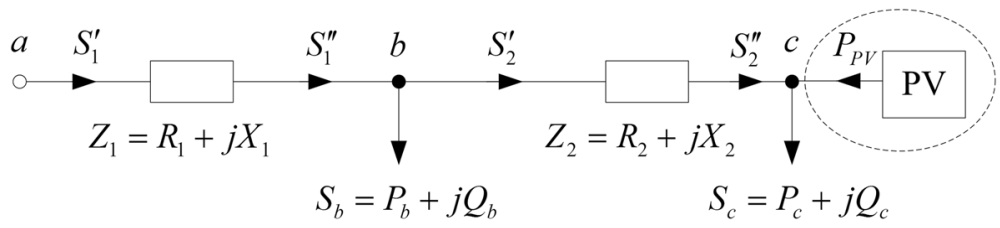

Fig. 1 Three-node network 
reactive power of the PV plant $Q_{P V}$ and $Q_{c}$ are both zero, active power $P_{P V}$ is larger than $P_{c}$.

We can see that PV plant is not only to meet the load demand of node $c$, but also provide power to the grid. $S_{2^{\prime \prime}}$ and $S_{2^{\prime}}$ are negative and have the opposite direction with original direction without PV. $S_{1^{\prime \prime}}$ and $S_{1^{\prime}}$ become smaller than before. Therefore, $\Delta V_{1}$ becomes smaller and $V_{b}$ becomes larger, $\Delta V_{2}$ becomes negative. The voltage of node $c$ is larger than node $b$.

From the above analysis, we can see that the voltage of node $c$ is larger than node $b$ after the access of PV plant. This is only a simple three-node system analysis. In fact, the number of network nodes and PV plants are more so that the situation of voltage is more complicated. The traditional way of voltage regulation, for example, the on-load tap changer, may lead to make a node to return to normal, while the other nodes are beyond the allowable range.

With the increasing configuration of DESSs on PV plants, the use of DESS for voltage regulation becomes a new regulated method. DESS can regulates voltage by a variety of ways, such as absorption or generation of active power, absorption or generation of reactive power, combination of active power and reactive power [1517].

\subsection{Proposed coordinated control strategy}

This paper presents a coordinated control method of voltage regulation using DESS. The flow cahrt is shown in the Fig. 2. First, according to the monitoring information of DESSs and nodes voltage, voltage state is judged if there are some node voltages beyond their limit. Secondly, judge whether the SoC is too high or low to use

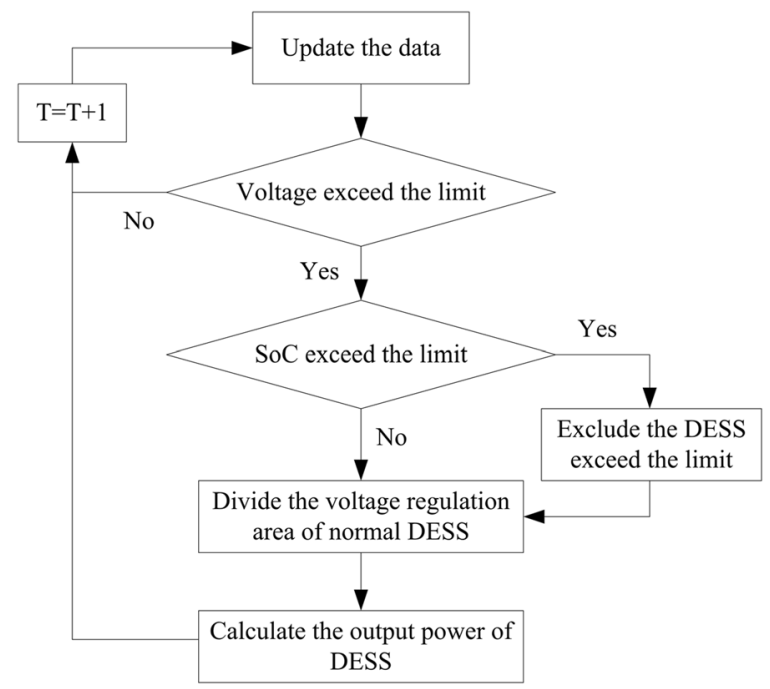

Fig. 2 The flow chart of the proposed strategy to regulate voltage, and exclude the DESS exceed the limit.Then, according to the impact degree of the output power of DESSs, the voltage regulation areas of normal DESSs are divided. The DESS plays an effective role in adjusting the node voltage in its own voltage regulation area, but the influence on the outside area is small. Finally, the action sequence of DESSs and the output power is determined.

\subsection{Division of voltage regulation area}

The distribution network usually contains a number of DESSs. If many DESSs act at the same time when a node voltage changes, this kind of disorder will not only play a good voltage regulator effect, but will deteriorate the voltage quality of other nodes. Therefore, for a distribution network with multiple DESSs, it is necessary to determine which nodes does the DESS have a greater effect on the voltage regulation. The voltage of these nodes is divided into an area. When the voltage of a node in this area is out of its range, the DESS in this area will act for voltage regulation.

The regulated area can be divided by calculating the variation of voltage caused by the change of output power of DESSs. Therefore, the power flow calculation method can be introduced. The $\mathrm{P}-\mathrm{Q}$ decomposition equation for the power flow calculation of complex power systems is

$$
\left[\begin{array}{c}
\Delta P \\
\Delta Q
\end{array}\right]=\left[\begin{array}{ll}
\frac{\partial P}{\partial \delta} & \frac{\partial P}{\partial V} \\
\frac{\partial Q}{\partial \delta} & \frac{\partial Q}{\partial V}
\end{array}\right]\left[\begin{array}{c}
\Delta \delta \\
\Delta V
\end{array}\right]
$$

This equation can be transformed to:

$$
\left[\begin{array}{c}
\Delta \delta \\
\Delta V
\end{array}\right]=[J]^{-1}\left[\begin{array}{c}
\Delta P \\
\Delta Q
\end{array}\right]=\left[\begin{array}{ll}
\frac{\partial P}{\partial \delta} & \frac{\partial P}{\partial V} \\
\frac{\partial Q}{\partial \delta} & \frac{\partial Q}{\partial V}
\end{array}\right]^{-1}\left[\begin{array}{c}
\Delta P \\
\Delta Q
\end{array}\right]
$$

$\Delta P$ and $\Delta Q$ are the output of active power and reactive power changes of DESS respectively. $\Delta V$ and $\Delta \delta$ are the voltage amplitude and phase angle changes respectively.

The inverse matrix of the Jacobian matrix is defined as the voltage sensitivity matrix, that is

$$
\left[V_{S}\right]=[J]^{-1}=\left[\begin{array}{ll}
\frac{\partial \delta}{\partial P} & \frac{\partial \delta}{\partial Q} \\
\frac{\partial V}{\partial P} & \frac{\partial V}{\partial Q}
\end{array}\right]
$$

$\left[\frac{\partial V}{\partial P}\right]$ is the voltage-active sensitivity matrix and $\left[\frac{\partial V}{\partial Q}\right]$ is the voltage-reactive power sensitivity matrix. They 
indicate respectively the amount of voltage change caused by the change of active and reactive power of DESS.

The voltage change of node $i$, caused by the active and reactive power disturbance of node $j$, is:

$$
\Delta V_{i}=\left[\frac{\partial V_{i}}{\partial P_{j}}\right] \Delta P_{j}+\left[\frac{\partial V_{i}}{\partial Q_{j}}\right] \Delta Q_{j}
$$

If the change of DESS is constant, the higher the voltage sensitivity, the stronger the support effect of the DESS on the voltage. In other words, the lower the voltage sensitivity, the weaker the support effect of the DESS on the voltage.

According to the concept of voltage sensitivity, the distribution network can be divided into several areas. Each area has a corresponding DESS. When the voltage is beyond the limit, the corresponding DESS will charge or discharge to adjust the voltage. This paper mainly uses the active power to adjust the voltage. The voltage change of can be expressed as:

$$
\Delta V_{i}=\left[\frac{\partial V_{i}}{\partial P_{j}}\right] \Delta P_{j}
$$

As described above, after removing the DESS that has not enough ability to regulate voltage, the division of the voltage regulation area is performed. The steps are as follows:

(1) Calculate the power flow based on the monitoring information. Obtain the Jacobian matrix of the distribution system and calculate the inverse matrix of Jacobian matrix.

(2) When the DESS $x$ act, $\Delta V_{i-x}$ can be obtained according to the formula (15). When the DESS $y$ acts, $\Delta V_{i-y}$ can be obtained according to the formula (15). Compare $\Delta V_{i-x}$ and $\Delta V_{i-y}$ if $\Delta V_{i-x}$ is larger, node $i$ belongs to the area of DESS $x$, where $x$ and $y$ are the mark number of DESSs. Every DESS charges or discharges to calculate the $\Delta V_{i}$, and all of them are compared to select the maximum.

(3) Since the Jacobian matrix is real-time and needs to be recalculated at each sampling time, the voltage regulation region also changes.

After dividing the voltage regulation area, it is also necessary to arrange the sequence of the operation of the DESS. The local DESS will act firstly, then the non-local DESS with higher $\Delta V_{i}$ will act. If there are non-local DESSs with same value of $\Delta V_{i}$, the one that has the closer distance to the root node will act.
Because root node is a slack node, the DESS closed to it has less impact on other nodes.

\subsection{Energy storage model and judgment of energy storage status.}

When the energy storage system is charged, the $\mathrm{SoC}$ is

$$
\operatorname{SoC}(t)=\operatorname{SoC}(t-\Delta t)+\frac{\eta_{c h} P \Delta t}{E_{\text {rate }}}
$$

When energy storage system discharge, the $\mathrm{SoC}$ is:

$$
\operatorname{SoC}(t)=\operatorname{SoC}(t-\Delta t)+\frac{\eta_{\text {disch }} P \Delta t}{E_{\text {rate }}}
$$

The SoC constraint is:

$$
S o C_{\min } \leq S o C(t) \leq S o C_{\max }
$$

Maximum power constraint for charge and discharge is:

$$
|P| \leq P_{\max }
$$

$E_{\text {rate }}$ is the rated capacity of energy storage. $\eta_{c h}$ and $\eta_{\text {disch }}$ are the energy storage charge and discharge efficiency, respectively. $\Delta E=P \Delta t$ is the energy charge or discharge energy. $P$ is the charge or discharge power. $\Delta t$ is the sampling time.

As mentioned earlier, we needs to determine whether there is distributed storage cannot participate in voltage regulation. This step is very important. If a DESS cannot respond to voltage regulation needs, but the system give a command to act, the DESS maybe break down.

The method to determine whether the energy storage can participate in voltage regulation is as follows:

(1) It is assumed that the voltage of the node i exceeds the minimum value, then the energy storage should be discharged. If the discharge power of corresponding DESS is rated power, it cannot increase more discharge power. If not, step (2) will be executed.

(2) Determine whether the SoC is the minimum, if so, then exclude the energy storage, if not, then the calculate the Jacobian matrix;

(3) The power flow will be calculated considering the charge or discharge power with the interval of $5 \mathrm{~kW}$ until find the minimum power value to make the voltage normal.

(4) When the voltage at node $i$ exceeds the maximum value, the judgment of the energy storage state is similar. 


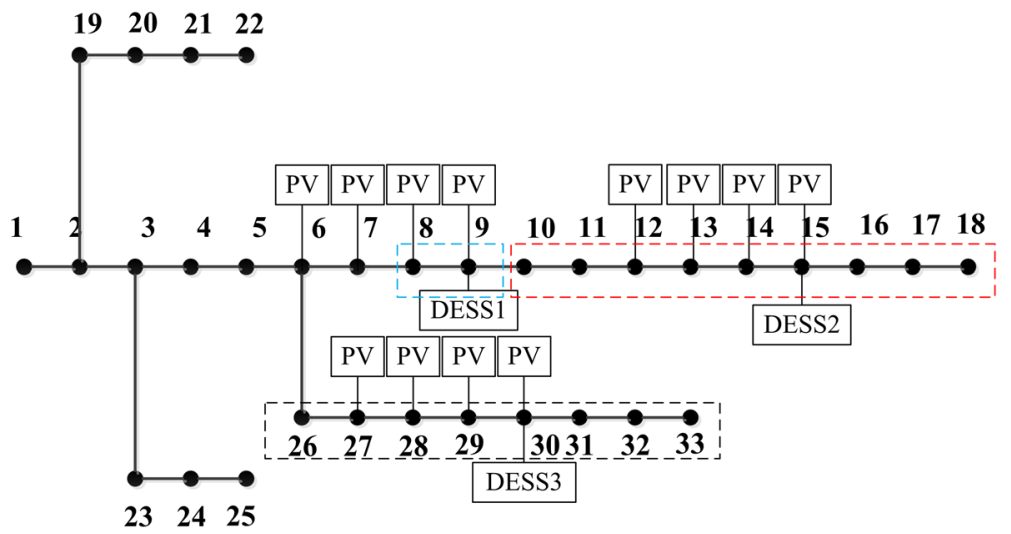

Fig. 3 IEEE 33-bus distribution system

\subsection{Case study}

The IEEE 33-bus distribution system [18] is used to simulation analysis, as shown in the Fig. 3. The load curve [19], PV generation scaling factors curve are as shown in Fig. 4. The maximum value of curve is 1 and using the load and PV value multiply by the scaling curve are the load and PV curves. In $10 \mathrm{kV}$ distribution system, the limit error of voltage is $\pm 7 \%$.

\subsubsection{Voltage analysis of the system without energy storage} As shown in Fig. 5, it is assumed that the voltage of the start node is 1.04 p.u.. In order to be more intuitive, we selected several typical curves. When the photovoltaic power station is not installed, from 8:00 to 22:00, the voltage of some nodes are lower than the 0.93 p.u.. It needs to take measures to solve this problem. Taking OLTC for instance, when the voltage of start node is regulated to 1.06 p.u., the voltage curve is got as shown

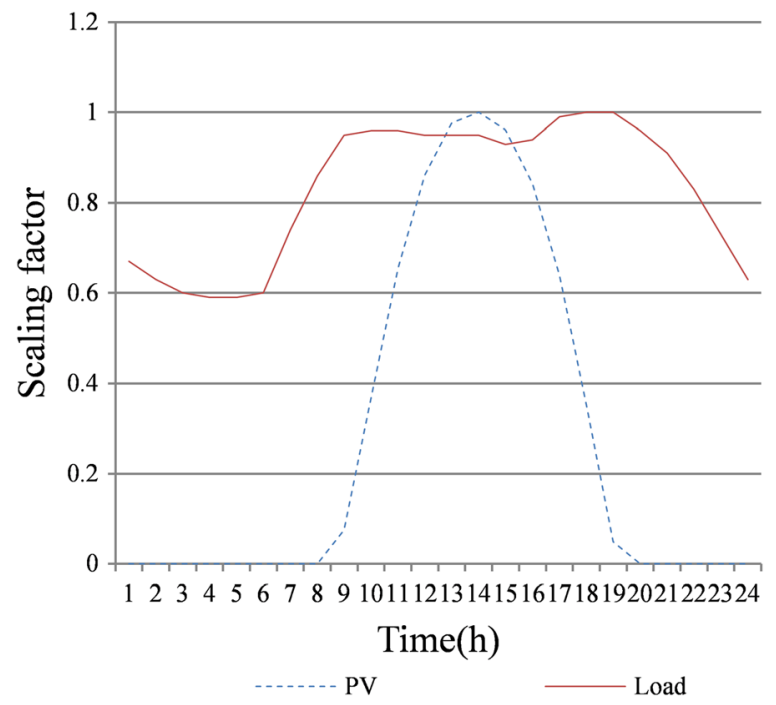

Fig. 4 The scaling factors of time-variant load and PV in Fig. 6. The smallest voltage of whole system is 0.932p.u. at node 33 on 19:00, so all of node voltage are in allowed range.

Four 500-kW PV plants are installed at node 6 9. Four 300-kW PV plants are installed at node 12 15 . Four 200-kW PV plants are installed at node $27 \sim 30$. Figure 7 shows the voltage curves of system with PV plants. From 13:00 to 15:00, the voltage of nodes 10 18 exceed the highest limit. In this period, the power of PV is so high that the node loads near the PV plants cannot consume. From 8:00 to 9:00, the nodes far from the start node exceed the lowest voltage limit. The same situation happens from 19:00 to 22:00. If OLTC still is used to regulate voltage in this kind of situation, it will act three times per day in this case.If there are more PV plants or other renewable power stations, the voltage of distribution network will be more complicated and the action of

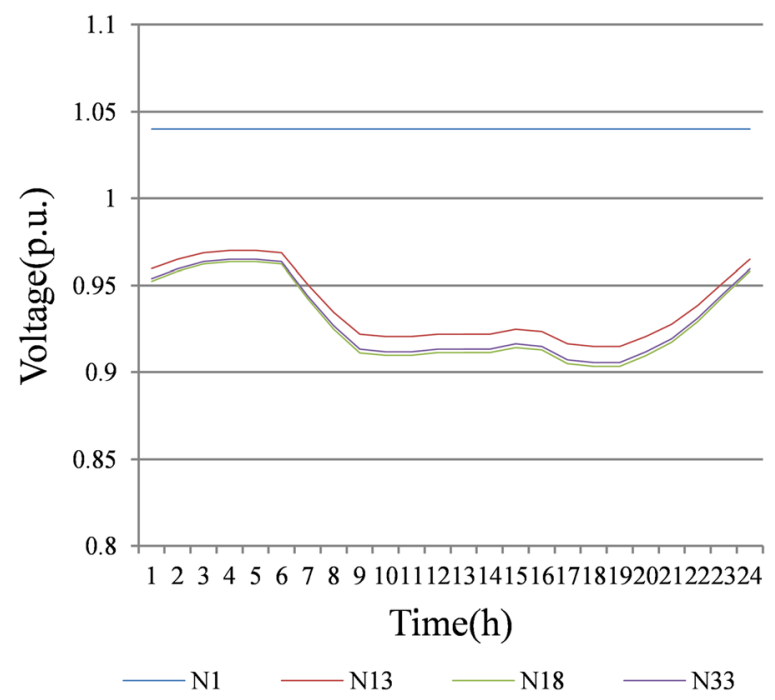

Fig. 5 Voltage profile with no PV and DESS 


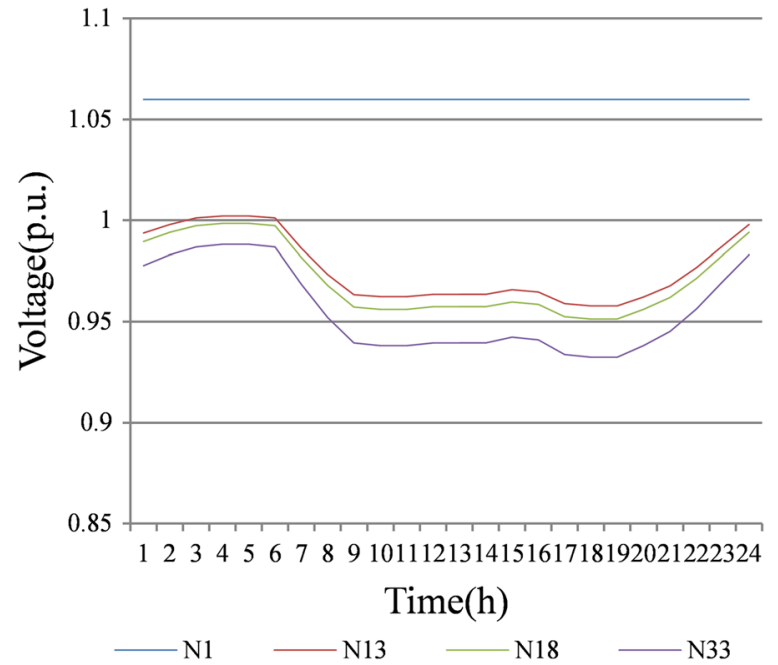

Fig. 6 Voltage profile after regulating by OLTC

OLTC will be more frequent. This will decrease the life of OLTC and increase the maintenance costs. The DESSs are installed at different nodes, which can regulate the voltage of nodes near itself.

\subsubsection{Voltage analysis of the system with energy storage}

Two $300 \mathrm{~kW} / 600 \mathrm{kWh}$ DESS are installed at node 9 and 15. They are DESS 1 and 2 respectively. One $300 \mathrm{~kW} / 800 \mathrm{kWh}$ DESS is installed at node 30 . It is DESS 3. After the DESSs are installed, the next step is to divide the voltage regulated area. Each DESS will have obvious effect on the nodes its own area.

Taking the situation of 14:00 for instance, the voltage curves are as shown in Fig. 8. Each curve of Fig. 8 is situation that only one DESS act with rating power. The node 8 and 9 are effect by DESS 1 more

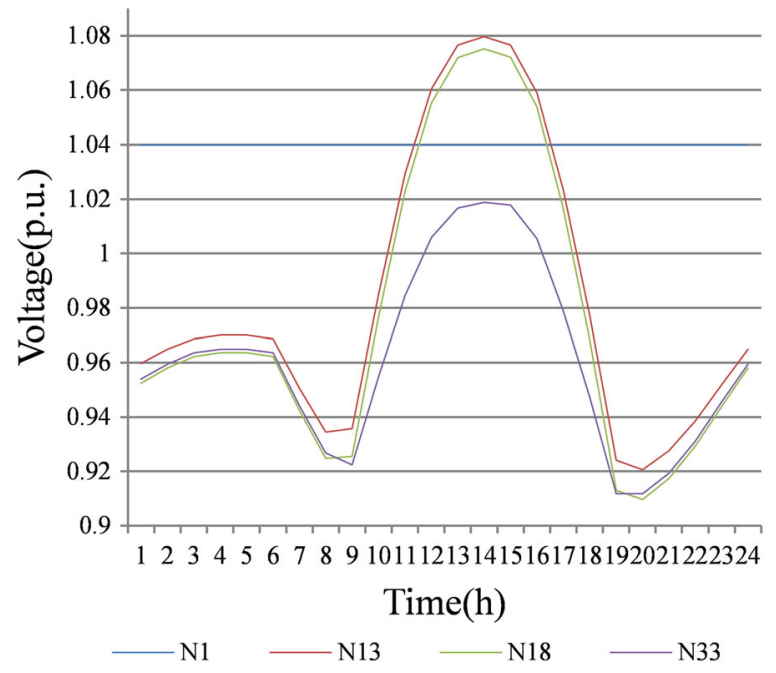

Fig. 7 Voltage profile with PV plants

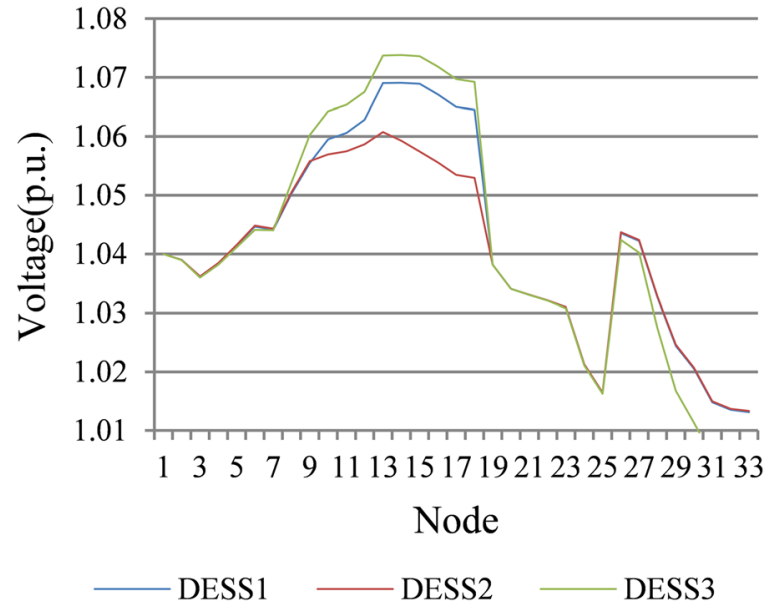

Fig. 8 Voltage profile of different DESS with rating output power at 14:00

obviously. Because the value of curve of DESS1is smaller than the value of DESS2, but the difference value is small. The curve of DESS 2 at node 10 18 is below the other curves, that is $\Delta V$ caused by DESS 2 is bigger. Therefore, the node $10 \sim 18$ belong to the area of DESS 2. The node $26 \sim 33$ are effected by DESS 3 more obviously. Other nodes are effect by three DESSs similarly. It can be judged that the regulation is more effective on the nodes near the DESS. The areas are shown in Fig. 3.

According to the division of the voltage,the size of the output power are calculated then. Still taking 14:00 for instance, the voltage of node 14 is up to $1.0798 \mathrm{pu}$. Obviously, if the voltage is controlled below $1.07 \mathrm{pu}$, the other nodes will return to the normal range. As shown in

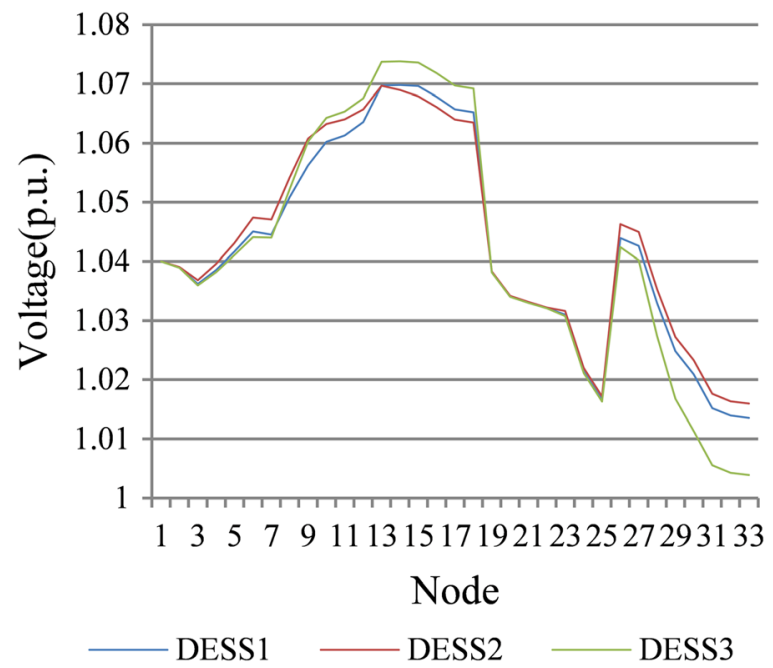

Fig. 9 Voltage profile of different DESS with different output power at $14: 00$ 
Fig. 9, the voltage of node 14 will be returned to normal range when the DESS1 charges at $280 \mathrm{~kW}$. For DESS 2, this power is only $160 \mathrm{~kW}$. However, the DESS 3 is so far from node 14 that it cannot restore the voltage even at maximum power. The output power of DESS is obtained by taking sample point every $5 \mathrm{~kW}$. It can be seen that the division of the voltage regulation area is very necessary. The DESS has little effect on the other voltage regulation area.

At 8:00, $15 \sim 18$ and $31 \sim 33$ nodes appear low voltage situation. The DESS 2 and 3 need to discharge to supply the load demand. AS shown in Fig. 10, curve a is the situation that only DESS 2 discharges at $60 \mathrm{~kW}$. Curve b is the situation that only DESS 3 discharges at $60 \mathrm{~kW}$. Curve $\mathrm{c}$ is the situation that DESS 2 and 3 both discharge at $60 \mathrm{~kW}$. Curve $\mathrm{c}$ is the situation that DESS 2 and 3 both discharge at $60 \mathrm{~kW}$. Curve $\mathrm{d}$ is the situation that DESS 2 discharges at $45 \mathrm{~kW}$ and 3 discharges at $40 \mathrm{~kW}$. In curve a and b, the nodes at DESS's own area return the normal voltage range. Compared curve $c$ and $\mathrm{d}$, we can see that two energy storage system output effect is greater than one.

As shown in Fig. 11, the curves are the SoC of three DESSs respectively. The curves of Fig. 12 are the active power of three DESSs respectively. We set the initial SoC values are 33\%, 33\% and 50\% respectively. At 8:00 and 9:00, DESS 1 and 2 discharge to support the voltage. At 13:00 15:00, DESS 2 charges to decrease the voltage. In this period DESS should be charged too because of the peak load in the evening. At 19:00 21:00, DESS 2 and 3 discharge to supply the load demand. However, at 21:00 and 22:00, the SoC of DESS 3 isn't insufficient, so the DESS 2 closed to DESS3 discharges to support the voltage of area 3.

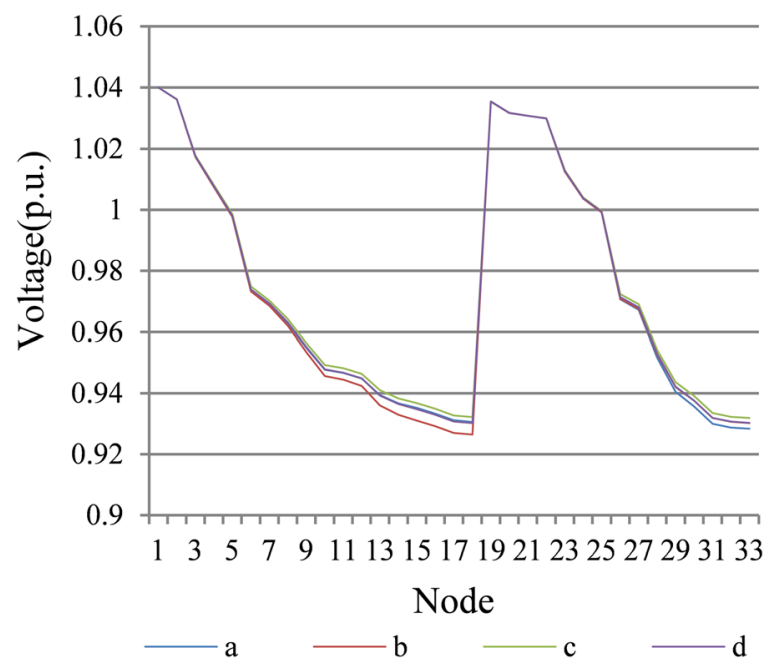

Fig. 10 Voltage profile of different DESS with different output power at 14:00 at 8:00

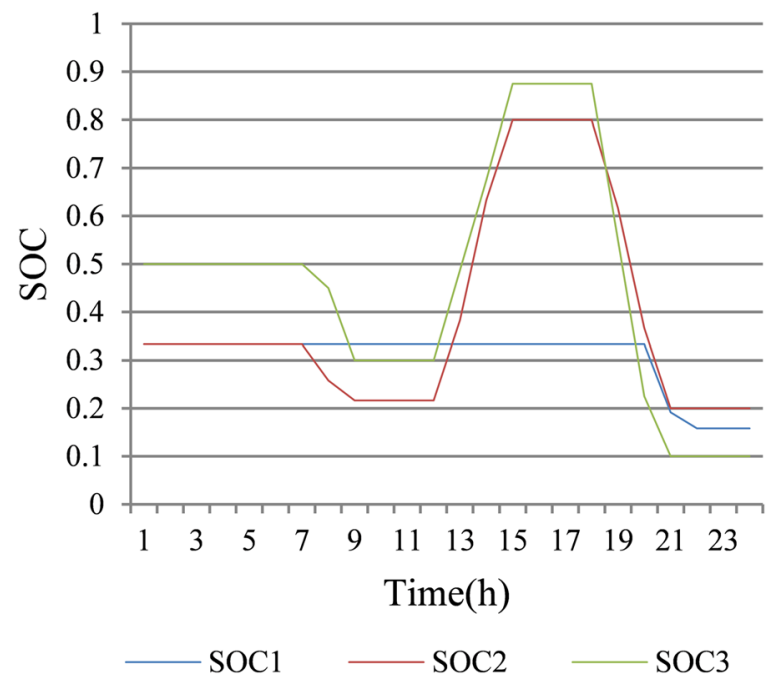

Fig. 11 The SoC curves of DESS

In the above simulation analysis, the voltage regulation area can be obtained by calculating the $\Delta V$ caused by the output power change of DESS. When the voltages of some nodes exceed the limit, but the SoC of corresponding DESS is not enough, the other DESS will be coordinated to act. The voltage will be returned to the normal range, although the non-local DESS will charge or discharge more power than the local DESS. In these simulations, we can see that the voltage can be regulated use DESSs. When the voltage is lower or higher than the limit value, DESSs will charge or discharge power according to the different voltage regulated areas and the different values exceeded the normal value. When the SoC of local DESS exceeds the allowed range or is not enough, the non-local DESS will coordinately act to regulate voltage.

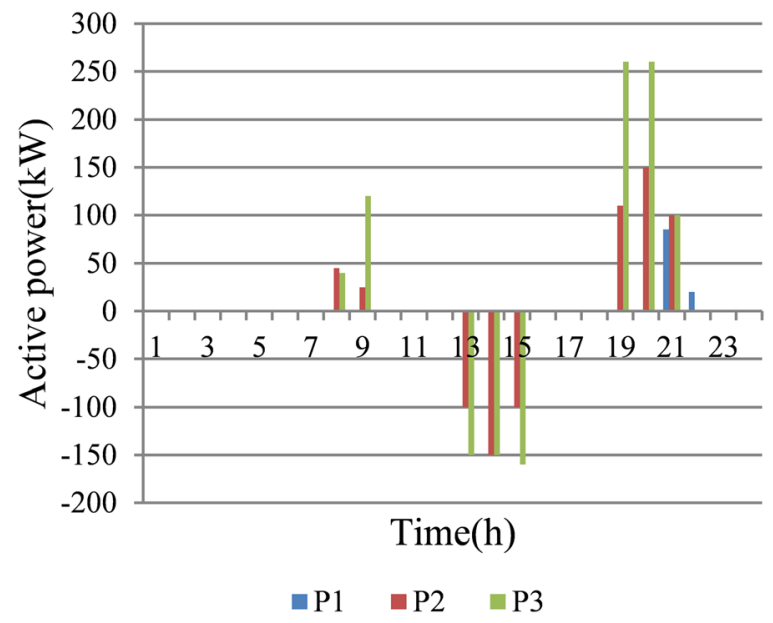

Fig. 12 The active power of DESS 


\section{Conclusions}

The traditional methods of voltage regulation are not suitable for active distribution system because of the access of PV plant. This paper presents a method of voltage regulation by DESS. The main conclusions are as follows:

(1) The voltage will become more disordered if more PV stations access the distribution network.

(2) The proposed method is able to divide the voltage regulation areas. The DESSs can charge or discharge when the voltage of their own area exceed the limit.

(3) If the voltages of two or more areas exceed the limitation at the same time, the DESSs will act. The effect of two DESSs is larger than one. Taking 8:00 for instance, DESS 2 and 3 discharge respectively $60 \mathrm{~kW}$ to make the voltage of their own area back to the normal value, while $45 \mathrm{~kW}$ and $40 \mathrm{~kW}$ discharged by DESS2 and 3 can achieve this effect when they act both.

(4) When the voltages of some nodes exceed the limit, but the SoC of corresponding DESS is not enough, the other DESS can be coordinated to act. And it can achieve the regulation effect too.

The proposed coordinated control strategy is suitable for medium voltage distribution network or larger system. The future work will deal with adapting active and reactive power to regulate voltage and optimize the output power of multiple DESSs.

\section{Acknowledgements}

Not applicable.

\section{Funding}

This paper is supported by The National Key Research and Development Plan, Energy Storage Technology of 10MW Level Redox Battery, 2017 YFB0903504.

\section{Availability of data and materials}

Not applicable.

\section{Authors' contributions}

DZ contributed significantly to analysis, manuscript preparation and manuscript submission as a corresponding author; $J \mathrm{~L}$ and DH helped perform the study analysis with constructive discussions, professional advice and revised the manuscript. All authors read and approved the final manuscript.

\section{Ethics approval and consent to participate}

Not applicable.

\section{Consent for publication}

Not applicable.

\section{Competing interests}

The authors declare that they have no competing interests.

\section{Author details}

${ }^{1}$ China Electric Power Research Institute, Haidian District, Beijing 100192. China. ${ }^{2}$ State Key Laboratory of Operation and Control of Renewable Energy \& Storage System, China Electric Power Research Institute, Haidian District, Beijing 100192, China.
Received: 24 May 2017 Accepted: 10 January 2018

Published online: 26 January 2018

\section{References}

1. Wang, L., Liang, D. H., Crossland, A. F., Taylor, P. C., Jones, D., \& Wade, N. S. (2015). Coordination of multiple energy storage units in a lowvoltage distribution network. IEEE Transactions Smart Grid, 6(6), 1-1.

2. Todorovski, M. (2014). Transformer voltage regulation-Compact expression dependent on tap position and primary/secondary voltage. IEEE Transactions Power Delivery, 29(3), 1516-1517.

3. Masters, C. L. (2002). Voltage rise: The big issue when connecting embedded generation to long $11 \mathrm{kv}$ overhead lines. Power Engineer, 16(1), 5-12.

4. Xingtian, F., Tongzhen, W., \& Lingzhi, K. (2010). Influence of high permeability distributed generation on voltage quality of distribution network. Water Resources Power, 28(9), 154-157.

5. Shibo, L. (2013). Research on distribution network voltage regulation strategy adapting to distributed power supply access. Shandong: Shandong University of Technology.

6. Tonkoski, R., Lopes, L. A. C., \& El-Fouly, T. H. M. (2010). Droop-based active power curtailment for overvoltage prevention in grid connected PV inverters. IEEE Int Symposium Industrial Electronics (pp. 2388-2393). IEEE.

7. Jacxsens, M., Meersman, B., Vandoorn, T. L., Kooning, J. D. M. D., \& Vandevelde, L. (2011). Overview of voltage control strategies in medium voltage networks with implementation of distributed generation. Renewable Power Generation (pp.1-6). IET.

8. Tingru M, Guibin Z, Chunhua X, Weijie S, Yuwei M. (2017). A Voltage Regulation Method Based on District-dividing Coordinated Control for Active Distribution Network. Proceedings of the CSEE, 37(10),2852-2860.

9. Marra, F., Yang, G., Traeholt, C., Ostergaard, J., \& Larsen, E. (2014). A decentralized storage strategy for residential feeders with photovoltaics. IEEE Transactions Smart Grid, 5(2), 974-981.

10. Kabir, M. N., Mishra, Y., Ledwich, G., Dong, Z. Y., \& Wong, K. P. (2014). Coordinated control of grid-connected photovoltaic reactive power and battery energy storage systems to improve the voltage profile of a residential distribution feeder. IEEE Transactions Industrial Informatics, 10(2), 967-977.

11. Brenna, M., Berardinis, E. D., Carpini, L. D., Foiadelli, F., Paulon, P., Petroni, P., et al. (2013). Automatic distributed voltage control algorithm in smart grids applications. IEEE Transactions Smart Grid, 4(2), 877-885

12. Kashem, M. A., \& Ledwich, G. (2005). Multiple distributed generators for distribution feeder voltage support. IEEE Transactions on Energy Conversion 20(3), 676-684.

13. Wang, Y., Tan, K. T., Peng, X. Y., \& So, P. L. (2016). Coordinated control of distributed energy-storage systems for voltage regulation in distribution networks. IEEE Transactions Power Delivery, 31(3), 1132-1141.

14. Zeraati, M., Golshan, M. E. H., \& Guerrero, J. (2017). Distributed control of battery energy storage systems for voltage regulation in distribution networks with high pv penetration. IEEE Transactions Smart Grid, 99, 1.

15. Avilés, D. A., Guinjoan, F., Barricarte, J., Marroyo, L., Sanchis, P., \& Valderrama, H. (2012). Battery management fuzzy control for a grid- tied microgrid with renewable generation (Vol. 2, pp. 5607-5612). IECON 2012 -, Conference on IEEE Industrial Electronics Society IEEE.

16. Silva-Monroy, C. A., \& Watson, J. P. (2014). Integrating energy storage devices into market management systems. Proceedings of the IEEE, 102(7), 1084-1093.

17. Hill, C. A., Such, M. C., Chen, D., Gonzalez, J., \& Grady, W. M. (2012). Battery energy storage for enabling integration of distributed solar power generation. IEEE Transactions on Smart Grid, 3(2), 850-857.

18. Venkatesh, B., Ranjan, R., \& Gooi, H. B. (2004). Optimal reconfiguration of radial distribution systems to maximize loadability. IEEE Transactions on Power Systems, 19(1), 260-266.

19. Atwa, Y. M., El-Saadany, E. F., Salama, M. M. A., Seethapathy, R., Assam, M., \& Conti, S. (2011). Adequacy evaluation of distribution system including wind/solar dg during different modes of operation. IEEE Transactions on Power Systems, 26(4), 1945-1952. 